\title{
Delayed complications after myocardial contusion
}

\author{
E D Grech, P A Dodds, R A Perry
}

\begin{abstract}
A 45 year old farmer was kicked in the chest by a horse. In the days following the injury episodic breathlessness developed and he was admitted to hospital with right ventricular failure and pulmonary emboli. Echocardiography showed global right ventricular dysfunction but a right ventricular mural thrombus, the likely source of the pulmonary emboli, was not seen. He gradually recovered after treatment with anticoagulant. One month later he presented with a further complicationcomplete atrioventricular dissociationthat required a dual chamber pacemaker implantation.

This patient had few initial manifestations of right ventricular myocardial contusion and this case illustrates that such patients should be closely monitored for delayed complications.
\end{abstract}

\section{(Br Heart J 1992;68:529-30)}

Non-penetrating injuries often cause myocardial contusion, the reported incidence ranges from $8 \%^{1}$ to $76 \%^{2}$ and reflects the variable criteria used. Because the right heart is nearer the sternum it is more often affected than the left. Motor vehicle accidents, in particular steering wheel injuries, ${ }^{3}$ are the commonest cause. Others include falls from heights, sports accidents, and animal kicks. ${ }^{4}$ Myocardial contusion can occur in isolation but it is more frequently associated with multiple injuries. Early complications, often the immediate effects of mechanical dysfunction and tachycardia, usually resolve. However, delayed secondary complications are not only unpredictable but may become life-threatening if unrecognised. This case report illustrates these late problems, which arose despite few clinical signs during the initial presentation.

\section{Case report}

A previously fit 45 year old farmer presented to the casualty department of a nearby district general hospital with severe chest pain. He had received a direct blow to the sternum and ribs after being kicked by the hind leg of a horse. Examination showed tenderness and minor superficial skin lacerations in this area. He was haemodynamically stable. Air in the soft tissues was attributed to the skin lacerations. There was a small right basal pleural reaction but chest and rib $x$ rays showed no sternal or rib fractures. The skin grazes were cleaned and dressed, and oral dihydrocodeine was prescribed before the patient was discharged.

Two days later he returned to the same casualty department with continuing sternal chest pain, which had not responded to dihydrocodeine, and mild ankle swelling. Despite stronger analgesia his non-cardiac chest pain remained troublesome and in addition he began to experience paroxysms of severe breathlessness. When he re-attended one week later he was dyspnoeic at rest and was admitted. He was pyrexial $\left(38^{\circ} \mathrm{C}\right)$ with tachycardia of 140 per minute. The jugular venous pressure was raised to ear level and his blood pressure was $120 / 70 \mathrm{~mm} \mathrm{Hg}$. A gallop rhythm was audible and chest auscultation showed scattered bilateral basal crepitations with reduced left basal air entry. Sacral oedema and gross ankle oedema were present. His blood count was normal apart from a raised white cell count of $14.6 \times 10^{9} / 1(83 \%$ neutrophils). Chest $x$ ray showed bilateral basal pleural effusions, a considerable increase in the prominence of hilar vascular shadows and an enlarged cardiac silhouette (though this was not globular). An electrocardiogram showed sinus tachycardia (130 beats per minute), a right bundle branch block pattern, and an inferior infarct pattern. Despite treatment with intravenous diuretic his condition deteriorated. He became hypoxic $\left(\mathrm{Po}_{2} 6 \cdot 7 \mathrm{kPa}\right.$ in air) and confused, although he remained normotensive $(130 / 70 \mathrm{~mm} \mathrm{Hg}$ ) and did not require inotropic support. At this time he was referred to the regional cardiac unit. A transthoracic echocardiogram showed severe global right ventricular dysfunction, less pronounced in the septal region with no clear evidence of thrombus, and normal left ventricular contractility. No valve or pericardial disease was detected. The patient declined a transoesophageal echocardiogram. Because the clinical picture was suggestive of pulmonary emboli a ventilation/perfusion scan was carried out. It showed multiple bilateral perfusion mismatches. After anticoagulation and with continued intensive treatment over the next 10 days he made a steady recovery and was discharged on warfarin.

At an outpatient clinic one month later he still had moderate breathlessness on exertion and lethargy. There was a bradycardia and an electrocardiogram showed complete nodal atrioventricular block with a ventricular rate of 44 per minute. Before dual chamber pacemaker implantation a transoesophageal 
echocardiogram was performed to look for residual thrombus. This confirmed an improvement in right ventricular contractility but no thrombus was seen. The patient's symptoms improved considerably after pacemaker implantation.

\section{Discussion}

In myocardial contusion there are histological changes of myocardial cell necrosis, erythrocyte extravasation, and leucocyte infiltration. ${ }^{5}$ Similarities with acute myocardial infarction have been reported ${ }^{6}$ and scar tissue formed during healing may account for some of the later complications. The clinical criteria for the diagnosis of myocardial contusion in the acute stage remain uncertain and the importance and significance of the diagnosis has given rise to some controversy. ${ }^{7}$ In a retrospective review of 524 patients admitted with suspected myocardial contusion Foil et al concluded that in patients with isolated chest wall injury all significant clinical sequelae would become apparent almost immediately after injury ${ }^{8}$ Our case report shows that this is not always so and unrecognised delayed sequelae such as pulmonary emboli or atrioventricular dissociation can be life-threatening. Our patient presented nine days after the injury with a combination of right ventricular dysfunction and recurrent multiple pulmonary emboli caused by right ventricular mural thrombus formation, a well-recognised complication of myocardial contusion. Timberlake and McSwain suggested that echocardiography should assess not only the extent of myocardial injury but should also be targeted to show evidence of mural thrombus. ${ }^{9}$ In our patient a transoesophageal echocardiogram was planned but initially declined by the patient.
Although arrhythmias and conduction disturbances are common soon after myocardial contusion these are usually transient and are almost certainly the direct result of injury. Delayed conduction disturbances have not been reported before. Because fibrosis and scar formation are part of the healing process of myocardial contusion, ${ }^{6}$ we postulate that in our patient this mechanism was a likely cause for the atrioventricular dissociation that ensued after a few weeks. The block at the nodal level suggests that the fibrotic process affected the septum in the region of the atrioventricular node or the intranodal portion of the common His bundle. Though the untreated course of this process is uncertain there was no reason at this stage to expect resolution and therefore a DDD mode pacemaker was implanted and it resulted in symptomatic improvement.

Even after an apparently trivial injury patients whose mechanism of injury may damage the myocardium should be closely monitored for a wide range of delayed complications that may present insidiously and may result in adverse outcome.

1 Frazee RC, Mucha P, Farnell MB, Miller FA. Objective evaluation of blunt cardiac trauma. J Trauma 1986;26: 510-20.

2 Sigler LH. Traumatic injury to the heart; incidence of its occurrence in 42 cases of severe accidental bodily injury. Am Heart J 1945;30:459-78.

3 Gunnar WP, Martin M, Smith RF, Manglano R, Resnick DJ, Lopez V, Barrett JA. The utility of cardiac evaluation in the haemodynamically stable patient with suspected myocardial contusion. Am Surgeon 1991;57:373-7.

4 Jackson DH, Murphy GW. Non-penetrating cardiac trauma. Mod Concep Cardiovasc Dis 1976;55:123-8.

5 Moritz AR, Atkins JP. Cardiac contusion: An experimenta and pathologic study. Arch Pathol 1938;25:445-62.

6 Leidtke AJ, DeMuth WE. Nonpenetrating cardiac injuries: A collective review. Am Heart J 1973;86:687-97.

7 Healy MA, Brown R, Fleizer D. Blunt cardiac injury: Is this diagnosis necessary? J Trauma 1990;30:137-46.

8 Foil MB, Mackersie RC, Furst SR, Davis JW, Swanson MS Hoyt DB, Shackford SR. Am J Surg 1990;160:638-43.

9 Timberlake GA, McSwain NE. Thromboembolism as a complication of myocardial contusion: a new capricious syndrome. J Trauma 1988;28:535-40. 